\title{
Comparison of the 9-Month Intrastent Condition and 30-Month Clinical Outcomes After Resolute Zotarolimus-Eluting Stent Implantation Between Standard-Duration and 1-Month Dual Antiplatelet Therapy Followed by Prasugrel Monotherapy
}

\author{
Tomoyo Hamana, MD; Takahiro Sawada, MD; Wataru Fujimoto, MD; \\ Tsuyoshi Osue, MD; Yoshiro Tsukiyama, MD; Kenzo Uzu, MD; \\ Tomofumi Takaya, MD; Yoshinori Yasaka, MD; Hiroya Kawai, MD
}

\begin{abstract}
Background: In this study we investigated the efficacy and safety of very short duration (1-month) dual antiplatelet therapy (DAPT) followed by prasugrel monotherapy. In particular, we compared intrastent conditions using optical coherence tomography (OCT) after second-generation drug-eluting stent implantation between standard-duration and 1-month DAPT followed by prasugrel monotherapy.

Methods and Results: Between May 2015 and February 2018, 120 consecutive patients who underwent elective Resolute zotarolimus-eluting stent implantation were enrolled and divided into those receiving standard-duration or 1-month (1M) DAPT followed by prasugrel monotherapy; 47 patients ( $n=55$ stents) and 46 patients ( $n=54$ stents) in the standard and $1 M$ groups, respectively, completed the protocol. The primary endpoint was the prevalence of abnormal intrastent tissue at the 9-month examination, as observed by OCT. The secondary endpoint was the presence of composite adverse events, including all-cause death, myocardial infarction, stent thrombosis, target lesion and vessel revascularization, and major and minor bleeding. The prevalence of abnormal intrastent tissue was similar between the standard and $1 \mathrm{M}$ groups ( $1.6 \%$ vs. $1.5 \%$, respectively; non-inferiority $\mathrm{P}<0.01)$. There was a tendency for fewer composite events in the $1 \mathrm{M}$ than standard group at the 30 -month follow-up examination (28.3\% vs. $44.7 \%$, respectively; $P=0.41$ ).
\end{abstract}

Conclusions: In conclusion, 1M DAPT followed by prasugrel monotherapy after second-generation drug-eluting stent implantation was not inferior to standard-duration DAPT in terms of intrastent thrombus formation and composite adverse events.

Key Words: Abnormal intrastent tissue; Dual antiplatelet therapy (DAPT); Optical coherence tomography

$\mathbf{T}$ he optimal duration of dual antiplatelet therapy (DAPT) after percutaneous coronary intervention (PCI) using a drug-eluting stent (DES) remains controversial. Longer DAPT (>12 months) has been shown to significantly reduce the risk of developing stent thrombosis and major adverse cardiovascular and cerebrovascular events (MACCE) compared with aspirin monotherapy., ${ }^{1,2}$ However, newer DES and optimal medical therapy have decreased the incidence of stent thrombosis. ${ }^{3}$ Undoubtedly, it is more important to avoid bleeding than the development of thrombotic events because the bleeding events are positively associated with an increased risk of mortality. ${ }^{4}$ In addition, as the number of patients with a high bleeding risk (HBR) increases with aging of the population, recent studies have recommended shortened DAPT duration., 5

Recently, short-term ( $\leq 3$ months) DAPT followed by $\mathrm{P} 2 \mathrm{Y}_{12}$ receptor antagonist monotherapy was shown to be effective. ${ }^{68}$ Prasugrel is a $\mathrm{P} 2 \mathrm{Y}_{12}$ receptor antagonist that provides more prompt, powerful, and consistent platelet inhibition than clopidogrel. ${ }^{9}$ In Japan, the DAPT regimen of aspirin and prasugrel therapy is common; however, no study has analyzed the outcomes of short-term DAPT followed by prasugrel monotherapy.

In this study we investigated the efficiency of very short $(\leq 1$ month [1M]) DAPT followed by monotherapy with the P2Y 12 receptor antagonist prasugrel compared with a standard duration of DAPT after implantation of a secondgeneration DES. We also investigated the validity of the

Received November 20, 2020; accepted November 20, 2020; J-STAGE Advance Publication released online December 23, 2020 Time for primary review: 1 day

Division of Cardiovascular Medicine, Hyogo Prefectural Himeji Cardiovascular Center, Himeji, Japan

Mailing address: Takahiro Sawada, MD, Division of Cardiovascular Medicine, Hyogo Prefectural Himeji Cardiovascular Center, 520 Saisho-Kou, Himeji 670-0981, Japan. E-mail: taktak319@yahoo.co.jp

All rights are reserved to the Japanese Circulation Society. For permissions, please e-mail: cr@j-circ.or.jp ISSN-2434-0790 


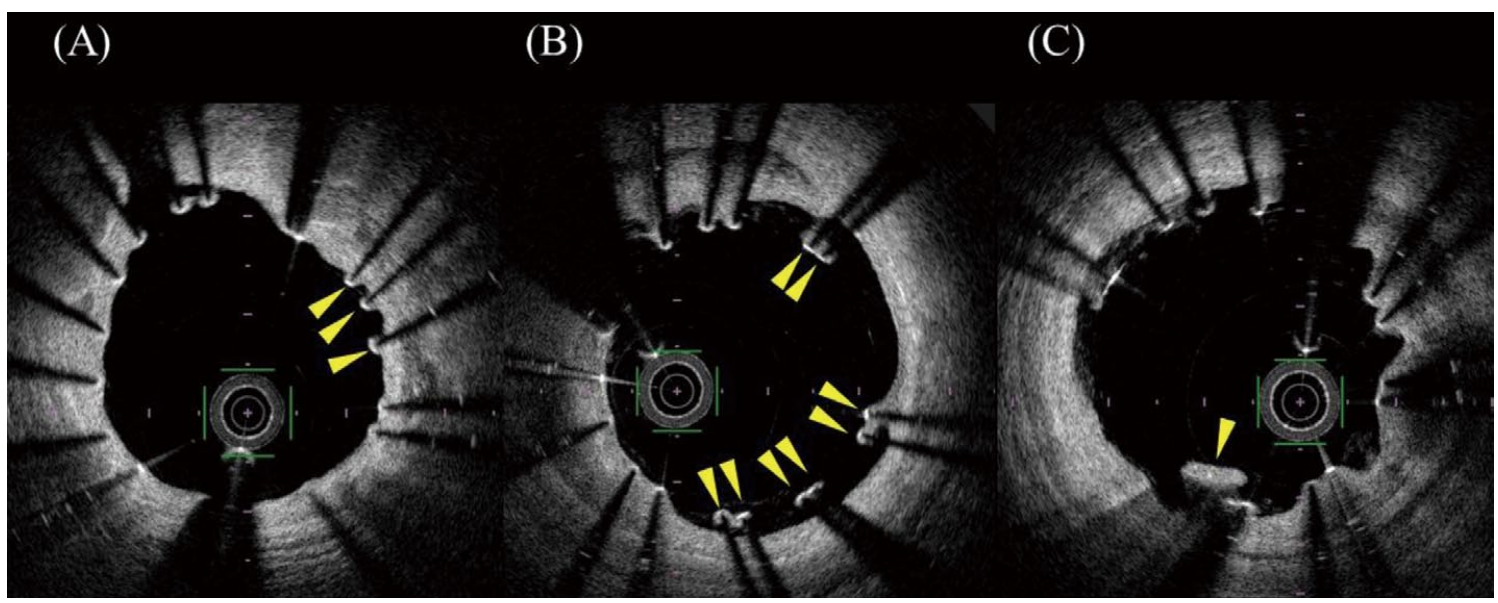

Figure 1. Optical coherence tomography findings. (A) Uncovered struts (arrowheads) are observed between 2 and 3 o'clock. (B) Malapposed struts (arrowheads) are observed between 1 and 6 o'clock. (C) Abnormal intrastent tissue (arrowhead) is observed at 7 o'clock.

regimen by observing mid-term vascular responses using optical coherence tomography (OCT).

\section{Methods}

\section{Study Population and Protocol}

This single-center prospective observational study included patients with angina pectoris who underwent PCI with the Resolute zotarolimus-eluting stent (R-ZES; Medtronic Cardiovascular, Santa Rosa, CA, USA; available according to the CE mark label of 1M DAPT for low-risk patients in Europe) and implantation by OCT guidance at the Hyogo Prefectural Himeji Cardiovascular Center between May 2015 and February 2018.

The inclusion criteria were age 20-90 years and a target lesion with significant stenosis $(>75 \%)$ by visual estimation and objective assessment of myocardial ischemia. The exclusion criteria were ejection fraction $<35 \%$, chronic renal failure with an estimated glomerular filtration rate $<30 \mathrm{~mL} / \mathrm{min} / 1.73 \mathrm{~m}^{2}$, acute coronary syndrome, allergy to the antiplatelet drug, left main disease and coronary bypass graft disease, bifurcation lesion requiring 2 stent implantations, and a stent with a diameter $\leq 2.25 \mathrm{~mm}$.

Just after the PCI procedure, the advantages and disadvantages of 1M DAPT followed by prasugrel monotherapy were explained to all patients except those requiring staged PCI. Patients who agreed to undergo the short-duration DAPT regimen were included in the $1 \mathrm{M}$ group; otherwise, patients were included in the standard-duration DAPT group. Patients who required staged PCI were included in the standard group because we could not unify the DAPT period to 1 month.

Aspirin (100mg/day) and prasugrel (3.75 mg/day) were prescribed at least 7 days prior to stent implantation; otherwise, a loading dose of prasugrel $(20 \mathrm{mg})$ was administered. In the $1 \mathrm{M}$ group, aspirin was stopped at 1 month after stent implantation and prasugrel was continued. In the standard group, DAPT with aspirin and prasugrel was continued until at least the 9-month follow-up coronary angiography examination. Subsequent antiplatelet therapy was administered at the discretion of the attending physician.

In all patients, stents were evaluated with OCT just after the index procedure and at 9 months after stent implantation. A clinical follow-up examination was performed at 30 months.

We evaluated the prediction of bleeding complications in patients undergoing stent implantation by calculating the PRECISE-DAPT ${ }^{10}$ and Pattern of Non-Adherence to Anti-Platelet Regimen in Stented Patients (PARIS) ${ }^{\mathbf{1 1}}$ scores to estimate the risk of developing ischemic and bleeding events after DES implantation.

Written informed consent was obtained from patients just after the index PCI procedure. The study protocol complied with the tenets of the Declaration of Helsinki and was approved by the Ethics Committee of the Hyogo Prefectural Himeji Cardiovascular Center (Reference no. 2015-13). This study was also registered in the UMIN Clinical Trials Registry (ID: UMIN000020421).

\section{Assessment of Platelet Reactivity}

Platelet reactivity was assessed just after stent implantation and at 1, 3, and 9 months after PCI using the VerifyNow P2Y 12 assay (Accumetrics, San Diego, CA, USA). The results are reported as $\mathrm{P} 2 \mathrm{Y}_{12}$ reaction units (PRU), which represent the amount of ADP-mediated aggregation specific to the platelet $\mathrm{P} 2 \mathrm{Y}_{12}$ receptor. Aspirin reaction units (ARU) were also measured using VerifyNow Aspirin assays (Accumetrics), which indicated arachidonic acid-induced platelet aggregation.

\section{Quantitative Coronary Angiography (QCA)}

Preprocedural, postprocedural, and 9-month follow-up QCA measurements were analyzed using an offline validated edge detection system (CMS; Medis Medical Imaging Systems, Leiden, Netherlands). Catheter calibration was used in all cases. Reference vessel diameter, minimum lumen diameter, percentage diameter stenosis, acute lumen gain, and late lumen loss were calculated. 


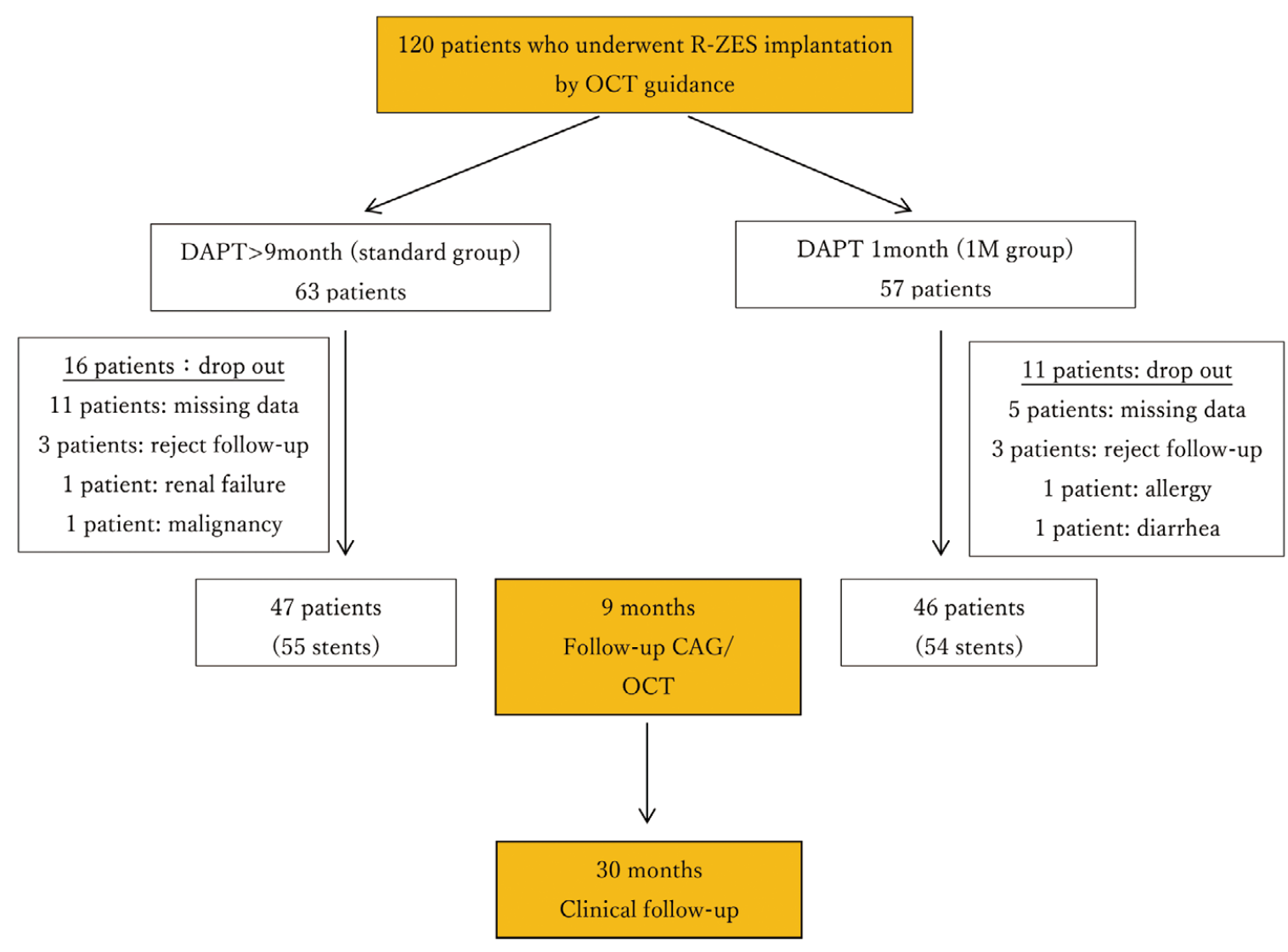

Figure 2. Patient flowchart. Of the 120 patients who underwent Resolute zotarolimus-eluting stent (R-ZES) implantation by optical coherence tomography (OCT) guidance, 63 patients were assigned to receive standard-duration dual antiplatelet therapy (DAPT) and 57 were assigned to receive the 1-month DAPT followed by prasugrel (1M). However, 16 and 11 patients in the standard and $1 \mathrm{M}$ groups, respectively, were excluded, leaving 47 patients ( $n=55$ stents) in the standard group and 46 patients $(n=54$ stents) in the $1 \mathrm{M}$ group. CAG, coronary angiography.

\section{OCT Imaging Acquisition and Analysis}

OCT images were acquired using an optical frequency domain imaging (OFDI) system (LUNAMAVE; Terumo, Tokyo, Japan) with a FastView imaging catheter or using an OCT system (ILUMIEN; Abbott Vascular, Santa Clara, CA, USA) with a Dragonfly imaging catheter (Abbott Vascular). As described previously, ${ }^{\mathbf{1 2}}$ blood in the lumen was replaced by contrast medium to acquire the OCT image. Intracoronary nitroglycerine $(100-300 \mu \mathrm{g})$ was administered before image acquisition and the pullback was at least $1 \mathrm{~cm}$ over the stent at a rate of $20 \mathrm{~mm} / \mathrm{s} .{ }^{12}$ All OCT images were reviewed using proprietary offline OCT imaging software by 3 experienced investigators (T.H., W.F., and T.O.) who were blinded to the angiographic data and clinical presentations. Cross-sectional OCT images were analyzed at 1-mm intervals.

Each OCT measurement was performed according to the methods described previously. ${ }^{\mathbf{1 2}}$ Briefly, neointimal thickness (NIT) was measured as the distance between the lumen surfaces of the neointima and the strut. The neointimal hyperplasia area was calculated as the stent area (lumen area) in each cross-sectional frame. Uncovered struts were defined as struts with a neointimal hyperplasia of $0 \mu \mathrm{m}$ (Figure 1A). Malapposed struts were defined as detachments from the vessel wall to the strut $\geq 117 \mu \mathrm{m}$ (Figure 1B), which was calculated by summing the values of the strut and polymer thickness and the estimated OCT resolution limit (i.e., $91+6+20 \mu \mathrm{m})$.

Intrastent thrombus and tissue protrusion were included as a single category, namely abnormal intrastent tissue (AIT), because sometimes they were difficult to identify by OCT. ${ }^{12}$ An AIT was defined as an irregular mass protruding over the stent strut into the lumen $(>100 \mu \mathrm{m}$; Figure 1C). The frequency of AITs observed was calculated as the number of frames, including any AIT, divided by the total number of cross-sectional frames.

\section{Study Endpoints}

The primary endpoint was the frequency of AITs observed at 9 months, because previous pathologic and clinical studies have suggested that the presence of AITs, such as an intrastent thrombus, is significantly associated with stent restenosis. ${ }^{13-15}$ The secondary endpoint was the composite of clinical adverse events, including all-cause death, myocardial infarction, stent thrombosis, target lesion revascularization (TLR), target vessel revascularization (TVR), and major or minor bleeding events during the 30 months after DES implantation. TLR was defined as 
repeated PCI or bypass graft placement for restenosis at the lesion of the index PCI site. TVR was defined as unplanned repeated PCI or bypass graft surgery because of stenosis of the same vessel treated at the index PCI. The severity of bleeding was assessed using Bleeding Academic Research Consortium (BARC) criteria. ${ }^{16}$ BARC Type 1 and 2 bleeding corresponded to minor bleeding, whereas BARC Type 3-5 bleeding was considered major bleeding. In particular, cases of major bleeding were considered those with a $>3.0 \mathrm{~g} / \mathrm{dL}$ decrement in the hemoglobin level, those requiring blood transfusion or surgical intervention, intracranial or intraocular bleeding, or fatal hemorrhage related to death.

\section{Statistical Analysis}

We calculated a sample size of 1,170 images per group, assuming a Type I error of 0.05 (1-sided), $80 \%$ power for the test, an assumed AIT detection rate of $1.5 \%$ (calculated based on preliminary data for the $1 \mathrm{M}$ group), a noninferiority margin of $1 \%$, and a $1: 1$ ratio for the sample size for the 2 groups. Because the unusable image rate was $10 \%$, we calculated that the optimal sample size would be 1,300 images per group in this study. The likelihood method was used to determine the significance of the non-inferiority test.

Continuous variables are presented as the mean \pm SD or as the median with interquartile range (IQR), and were compared using Student's t-test or the Mann-Whitney U-test for normally and non-normally distributed data, respectively. Categorical variables are presented as frequencies with percentages and were compared using the $\chi^{2}$ test or Fisher's exact test. An adverse events analysis was constructed using Kaplan-Meier estimates and compared using the log-rank test. Hazard ratios (HR) of bleeding events for the $1 \mathrm{M}$ group relative to the standard group with 2 -sided $95 \%$ confidence intervals (CIs) were calculated using the Cox proportional hazard model. All analyses were performed using MedCalc version 9.3 (MedCalc Software, Ostend, Belgium). For all tests, 2-sided $\mathrm{P}<0.05$ was considered significant.

\begin{tabular}{|c|c|c|c|}
\hline & Standard group & $1 \mathrm{M}$ group & $P$ value \\
\hline No. patients & 47 & 46 & \\
\hline Age (years) & $69.0 \pm 9.8$ & $68.2 \pm 9.2$ & 0.67 \\
\hline Male sex & $42(89.4)$ & $42(91.3)$ & 0.75 \\
\hline Body mass index $\left(\mathrm{kg} / \mathrm{m}^{2}\right)$ & $24.0 \pm 3.7$ & $23.6 \pm 2.4$ & 0.54 \\
\hline \multicolumn{4}{|l|}{ Coronary risk factors } \\
\hline Diabetes & $24(51.1)$ & $17(37.0)$ & 0.17 \\
\hline Dyslipidemia & $43(91.5)$ & $43(93.5)$ & 0.72 \\
\hline Hypertension & $42(89.4)$ & $43(93.5)$ & 0.48 \\
\hline Atrial fibrillation & $3(6.4)$ & $2(4.3)$ & 0.67 \\
\hline Cancer & $4(8.5)$ & $5(10.9)$ & 0.70 \\
\hline Prior MI & $6(12.8)$ & $7(15.2)$ & 0.73 \\
\hline Current smoker & $10(21.3)$ & $9(19.6)$ & 0.64 \\
\hline \multicolumn{4}{|l|}{ Laboratory data } \\
\hline Hemoglobin (g/dL) & $13.6 \pm 1.7$ & $13.8 \pm 1.4$ & 0.54 \\
\hline Albumin (g/dL) & $4.1 \pm 0.4$ & $4.1 \pm 0.4$ & 0.60 \\
\hline Creatinine (mg/dL) & $0.87 \pm 0.20$ & $0.80 \pm 0.16$ & 0.06 \\
\hline Blood urea nitrogen (mg/dL) & $16.9 \pm 5.1$ & $15.1 \pm 4.2$ & 0.07 \\
\hline Triglyceride (mg/dL) & 149 [103-212] & $144[91-218]$ & 0.75 \\
\hline LDL-C (mg/dL) & $101.2 \pm 30.5$ & $103.1 \pm 33.6$ & 0.78 \\
\hline $\mathrm{HbA1c}(\mathrm{g} / \mathrm{dL})$ & $6.5 \pm 0.9$ & $6.2 \pm 0.8$ & 0.05 \\
\hline BNP (pg/nL) & $30.1[16.9-81.4]$ & $32.9[16.4-55.0]$ & 0.65 \\
\hline \multicolumn{4}{|l|}{ Echocardiographic data } \\
\hline Ejection fraction (\%) & $56.1 \pm 8.9$ & $58.0 \pm 8.0$ & 0.29 \\
\hline \multicolumn{4}{|l|}{ Medication } \\
\hline Warfarin & $1(2.1)$ & $0(0.0)$ & 0.32 \\
\hline DOAC & $1(2.1)$ & $2(4.3)$ & 0.55 \\
\hline Statin & $44(93.6)$ & $42(91.3)$ & 0.67 \\
\hline ACEI/ARB & $33(70.2)$ & $23(50.0)$ & 0.048 \\
\hline$\beta$-blocker & $16(34.0)$ & $12(26.7)$ & 0.44 \\
\hline PPI & $46(97.9)$ & $42(91.3)$ & 0.16 \\
\hline CCB & $23(48.9)$ & $19(42.2)$ & 0.52 \\
\hline DPP-4 inhibiter & $16(34.0)$ & $13(28.9)$ & 0.57 \\
\hline Insulin & $5(10.6)$ & $0(0.0)$ & 0.023 \\
\hline
\end{tabular}

(Table 1 continued the next page.) 


\begin{tabular}{|c|c|c|c|}
\hline & Standard group & $1 \mathrm{M}$ group & $P$ value \\
\hline \multicolumn{4}{|l|}{ Platelet reaction units } \\
\hline At index procedure & $139.7 \pm 65.5$ & $128.6 \pm 72.3$ & 0.44 \\
\hline At 1 month & $157.1 \pm 55.2$ & $141.5 \pm 68.1$ & 0.24 \\
\hline At 3 months & $148.9 \pm 48.9$ & $131.9 \pm 60.2$ & 0.14 \\
\hline At 9-month follow-up & $158.7 \pm 44.7$ & $129.2 \pm 61.1$ & 0.01 \\
\hline \multicolumn{4}{|l|}{ Aspirin reaction units } \\
\hline At index procedure & $431.3 \pm 57.3$ & $432.7 \pm 58.5$ & 0.95 \\
\hline At 1 month & $483.0 \pm 73.6$ & $451.8 \pm 81.9$ & 0.21 \\
\hline At 3 months & $445.7 \pm 61.4$ & $596.8 \pm 50.9$ & $<0.001$ \\
\hline At 9-month follow-up & $465.7 \pm 83.9$ & $563.5 \pm 68.6$ & $<0.001$ \\
\hline PARIS thrombotic score & $3.0[1.3-4.0]$ & $1.0[0.0-3.0]$ & 0.0012 \\
\hline PARIS bleeding score & $7.0[4.0-8.8]$ & $6.0[4.0-8.0]$ & 0.38 \\
\hline PRECISE-DAPT score & $22.0[14.3-27.3]$ & $18.5[12.0-26.0]$ & 0.20 \\
\hline DAPT duration (days) & $498[306-1,095]$ & 30 [29-36] & $<0.001$ \\
\hline No. lesions & 55 & 54 & \\
\hline \multicolumn{4}{|l|}{ Target vessel } \\
\hline Left anterior descending artery & $18(32.7)$ & $23(42.6)$ & \\
\hline Left circumflex artery & $8(14.5)$ & $8(14.8)$ & \\
\hline Right coronary artery & $29(52.7)$ & $23(42.6)$ & 0.52 \\
\hline Type B2/C & $31(56.4)$ & $31(57.4)$ & 0.91 \\
\hline Use of rotablator & $3(5.6)$ & $5(9.4)$ & 0.45 \\
\hline Chronic total occlusion & $4(7.4)$ & $2(3.8)$ & 0.42 \\
\hline Stent diameter $(\mathrm{mm})$ & $3.09 \pm 0.45$ & $3.12 \pm 0.48$ & 0.72 \\
\hline Stent length (mm) & $21.1 \pm 6.4$ & $22.0 \pm 7.4$ & 0.66 \\
\hline Post-dilatation & $36(65.5)$ & $40(74.1)$ & 0.33 \\
\hline
\end{tabular}

Data are given as the mean $\pm \mathrm{SD}$, median [interquartile range], or $n(\%)$. ACEI, angiotensin-converting enzyme inhibitor; ARB, angiotensin receptor blocker; BNP, B-type natriuretic peptide; CCB, calcium channel blocker; DAPT, dual antiplatelet therapy; DOAC, direct oral anticoagulant; DPP-4, dipeptidyl peptidase-4; LDL-C, low density lipoprotein cholesterol; MI, myocardial infarction; PARIS, Pattern of Non-Adherence to Anti-Platelet Regimen in Stented Patients; PPI, proton pump inhibitor.

\section{Results}

\section{Patient Flow and Baseline Characteristics}

Of the 120 patients who underwent R-ZES implantation by OCT guidance, 63 and 57 were assigned to the standard and $1 \mathrm{M}$ groups, respectively. As shown in Figure 2, 16 and 11 patients in the standard and $1 \mathrm{M}$ groups, respectively, were excluded. This left 47 patients ( $\mathrm{n}=55$ stents) in the standard group and 46 patients $(n=54$ stents) in the $1 \mathrm{M}$ group.

Table 1 presents baseline patient and lesion characteristics. No significant differences were observed between the 2 groups in terms of baseline demographic factors, coronary risk factors, laboratory data, echocardiographic data, and medication, except for $\mathrm{HbAlc}$ values, the percentage of insulin usage, and the rate of prescription of angiotensinconverting enzyme inhibitors or angiotensin receptor blockers. Although the PARIS bleeding and PRECISEDAPT scores were similar between the 2 groups, the PARIS thrombotic score was higher in the standard than $1 \mathrm{M}$ group. In the standard group, the median DAPT duration was 498 days and $36.2 \%$ of the patients received DAPT during the 30-month follow-up period (Figure 3).

\section{Platelet Reactivity Assessment}

Table 1 and Figure 4 present comparisons between the PRU and ARU at the index procedure and at the 1-, 3-, and 9-month follow-up examinations. The measured PRU values were similar between the groups. However, although the ARU levels did not differ significantly between the groups at the index procedure and at the 1-month follow-up, they were significantly higher in the standard than $1 \mathrm{M}$ group at the 3- and 9-month follow-up examinations.

\section{QCA Measurements}

Table 2 shows the comparison of QCA measurements between the $1 \mathrm{M}$ and standard groups. The preintervention, post-stent implantation, and 9-month follow-up minimum lumen diameter, percentage stenosis area, acute gain, and late lumen loss were similar between the 2 groups.

\section{OCT Findings}

Table 2 shows the comparison of OCT findings between the $1 \mathrm{M}$ and standard groups at post-stent implantation and the 9-month follow-up. In all, 14,349 struts in 1,224 cross-sections were observed in the standard group and 14,581 struts in 1,301 cross-sections were observed in the $1 \mathrm{M}$ group.

Immediately after stent implantation and at the 9-month follow-up examination, the mean lumen and stent areas tended to be smaller in the $1 \mathrm{M}$ than standard group, but the differences did not reach statistical significance. The mean NIT at the 9-month follow-up examination was similar between the 2 groups. Although mean AIT area and the prevalence of AITs and malapposition were similar between the 2 groups immediately after PCI, the frequency of uncovered and malapposed struts was greater in the $1 \mathrm{M}$ than standard group. Nevertheless, the prevalence 


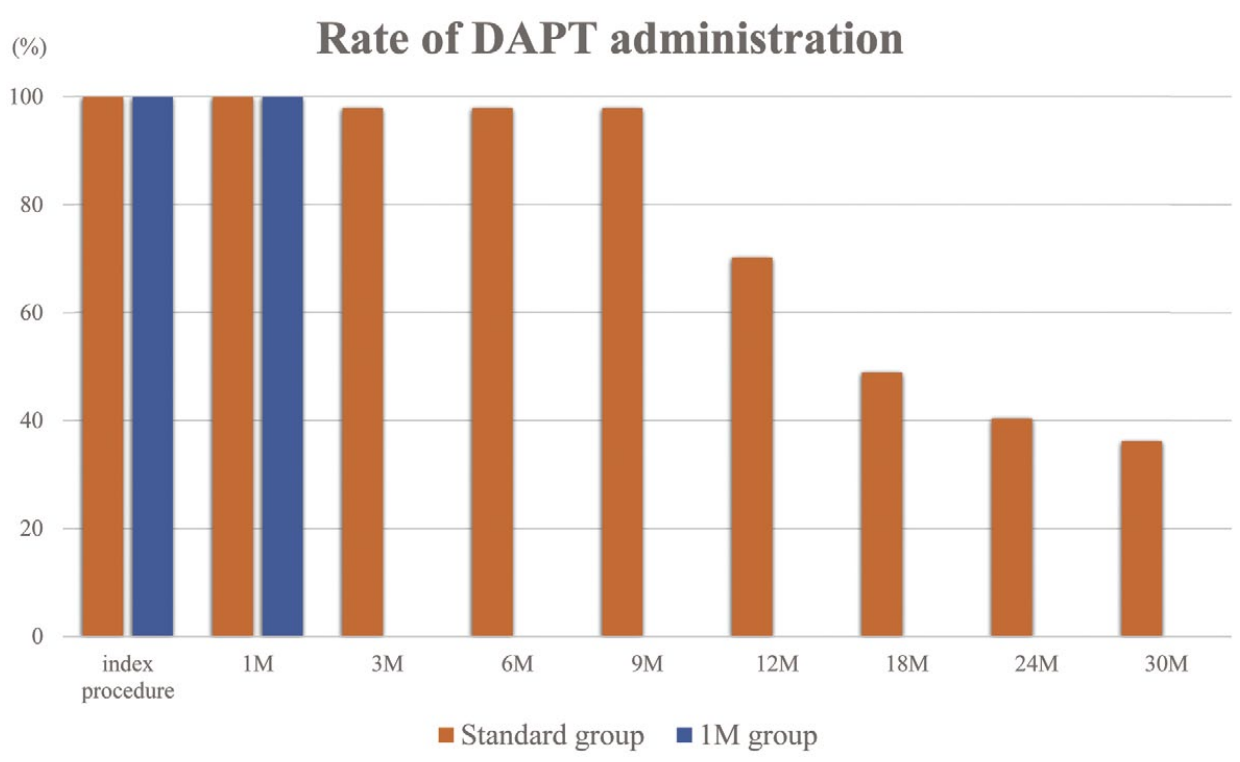

Figure 3. Comparison of the rate of dual antiplatelet therapy (DAPT) administration between patients receiving standard-duration DAPT and those receiving 1-month DAPT followed by prasugrel (1M). In the $1 \mathrm{M}$ group, aspirin was stopped at 1 month after stent implantation and prasugrel was continued. In the standard group, DAPT was continued until at least the 9-month follow-up coronary angiography examination. Subsequent antiplatelet therapy was administered at the discretion of the attending physician. Finally, $36.2 \%$ of the patients in the standard group received DAPT.

\section{(A)}

\section{P2Y12 Reaction Units}

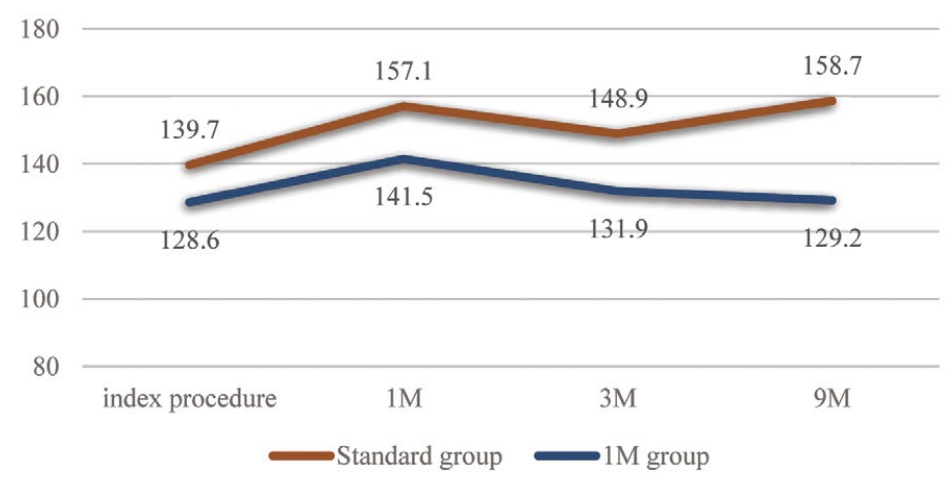

(B)

\section{Aspirin Reaction Units}

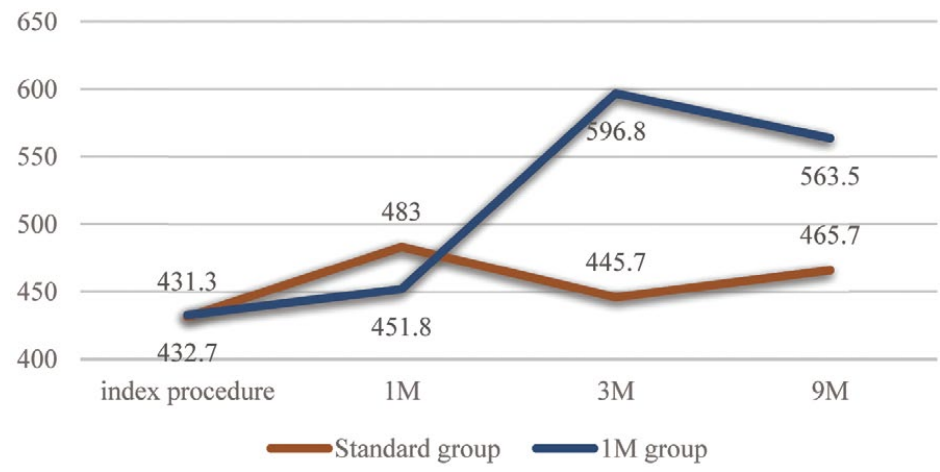

Figure 4. Comparison of $(\mathbf{A})$ platelet reaction units (PRU) and (B) aspirin reaction units (ARU) between patients receiving standard-duration dual antiplatelet therapy (DAPT) and those receiving 1-month DAPT followed by prasugrel $(1 \mathrm{M})$ at the index procedure and after 1,3 , and 9 months. 


\begin{tabular}{|c|c|c|c|}
\hline & $\begin{array}{c}\text { Standard group } \\
(n=55)\end{array}$ & $\begin{array}{c}\text { 1M group } \\
(n=54)\end{array}$ & $P$ value \\
\hline \multicolumn{4}{|l|}{ QCA data } \\
\hline \multicolumn{4}{|l|}{ Before interventions } \\
\hline Reference vessel diameter (mm) & $2.52 \pm 0.57$ & $2.56 \pm 0.60$ & 0.72 \\
\hline Minimum lumen diameter $(\mathrm{mm})$ & $0.99 \pm 0.50$ & $1.04 \pm 0.43$ & 0.59 \\
\hline$\%$ Area stenosis & $81.8 \pm 13.9$ & $81.6 \pm 11.7$ & 0.94 \\
\hline$\%$ Diameter stenosis & $61.9 \pm 19.3$ & $60.3 \pm 16.2$ & 0.64 \\
\hline \multicolumn{4}{|l|}{ After stent implantation } \\
\hline Reference vessel diameter (mm) & $3.21 \pm 0.45$ & $3.11 \pm 0.37$ & 0.24 \\
\hline Minimum lumen diameter $(\mathrm{mm})$ & $2.75 \pm 0.40$ & $2.72 \pm 0.38$ & 0.70 \\
\hline$\%$ Area stenosis & $25.7 \pm 11.7$ & $23.7 \pm 9.3$ & 0.34 \\
\hline$\%$ Diameter stenosis & $14.2 \pm 7.0$ & $12.8 \pm 5.5$ & 0.26 \\
\hline \multicolumn{4}{|l|}{ 9-month follow up } \\
\hline Reference vessel diameter (mm) & $3.04 \pm 0.49$ & $2.96 \pm 0.44$ & 0.38 \\
\hline Minimum lumen diameter (mm) & $2.57 \pm 0.48$ & $2.49 \pm 0.50$ & 0.45 \\
\hline$\%$ Area stenosis & $27.6 \pm 14.7$ & $28.3 \pm 14.4$ & 0.81 \\
\hline$\%$ Diameter stenosis & $15.5 \pm 9.8$ & $15.9 \pm 9.6$ & 0.84 \\
\hline Acute gain $(\mathrm{mm})$ & $1.73 \pm 0.58$ & $1.65 \pm 0.53$ & 0.47 \\
\hline Late lumen loss $(\mathrm{mm})$ & $0.18 \pm 0.53$ & $0.22 \pm 0.39$ & 0.63 \\
\hline \multicolumn{4}{|l|}{ OCT data } \\
\hline \multicolumn{4}{|l|}{ Cross-section based analysis } \\
\hline No. frames & 1,224 & 1,301 & \\
\hline \multicolumn{4}{|l|}{ After stent implantation } \\
\hline Mean lumen area $\left(\mathrm{mm}^{2}\right)$ & $7.73 \pm 2.12$ & $7.09 \pm 2.29$ & 0.15 \\
\hline Mean stent area $\left(\mathrm{mm}^{2}\right)$ & $7.86 \pm 2.07$ & $7.24 \pm 2.27$ & 0.16 \\
\hline Mean AIT area $\left(\mathrm{mm}^{2}\right)$ & $0.23 \pm 0.30$ & $0.23 \pm 0.26$ & 0.99 \\
\hline CS with AIT & $432(35.3)$ & 428 (32.9) & 0.20 \\
\hline CS with malapposition ( $\mathrm{n}, \%)$ & $169(13.8)$ & $178(13.7)$ & 0.93 \\
\hline \multicolumn{4}{|l|}{ 9-month follow-up } \\
\hline Mean lumen area $\left(\mathrm{mm}^{2}\right)$ & $7.67 \pm 2.03$ & $7.02 \pm 2.22$ & 0.13 \\
\hline Mean stent area $\left(\mathrm{mm}^{2}\right)$ & $6.97 \pm 1.98$ & $6.40 \pm 2.21$ & 0.18 \\
\hline Mean NIT area $\left(\mathrm{mm}^{2}\right)$ & $0.78 \pm 0.64$ & $0.68 \pm 0.4$ & 0.39 \\
\hline Mean malapposed area $\left(\mathrm{mm}^{2}\right)$ & $0.07 \pm 0.29$ & $0.07 \pm 0.24$ & 0.57 \\
\hline CS with AIT & $19(1.6)$ & $19(1.5)$ & 0.86 \\
\hline CS with uncovered strut & $166(13.6)$ & $215(16.7)$ & 0.03 \\
\hline CS with malapposition & $41(3.4)$ & $81(6.3)$ & 0.0007 \\
\hline \multicolumn{4}{|l|}{ Strut-based analysis at 9 months } \\
\hline No. struts & 14,349 & 14,581 & \\
\hline Mean NIT $(\mu \mathrm{m})$ & $80[50-150]$ & $80[50-140]$ & 0.60 \\
\hline Uncovered struts & $763(5.3)$ & $970(6.7)$ & $<0.0001$ \\
\hline Malapposed struts & $78(0.5)$ & $133(0.9)$ & 0.0002 \\
\hline
\end{tabular}

Data are given as the mean $\pm \mathrm{SD}$, median [interquartile range], or $\mathrm{n}(\%)$. AIT, abnormal intrastent tissue; CS, cross section; DAPT, dual antiplatelet therapy; NIT, neointimal thickness; OCT, optical coherence tomography; QCA, quantitative coronary angiography.

of AITs at the 9-month follow-up examination was similar between the standard and $1 \mathrm{M}$ groups $(1.6 \%$ and $1.5 \%$, respectively).

We set the non-inferiority margin for the prevalence of AITs at $1 \%$ and found that the upper limit of the 1 -sided $95 \% \mathrm{CI}$ for the difference between the $1 \mathrm{M}$ and standard groups was $<2.5 \%$ (difference $0.092 \%$; $95 \%$ CI -0.889 , 0.706 ). Therefore, non-inferiority of the $1 \mathrm{M}$ to standard group with respect to the prevalence of AITs was established (non-inferiority $\mathrm{P}<0.01$ ).

\section{Clinical Results}

Table 3 presents the clinical results. During the 30-month follow-up, there were no deaths and no myocardial infarction or stent thrombosis in the 2 groups. However, 5 and 1 patients in the standard and $1 \mathrm{M}$ groups, respectively, required TVR, and 4 and 1 patients, respectively, required TLR. Major bleeding was observed in 2 and 1 patients in the standard and $1 \mathrm{M}$ groups, respectively, whereas minor bleeding was observed in 14 and 11 patients, respectively. No significant intergroup difference was observed for any clinical event. Figure 5 presents the Kaplan-Meier analysis 


\begin{tabular}{|c|c|c|c|}
\hline & $\begin{array}{l}\text { Standard group } \\
(n=47)\end{array}$ & $\begin{array}{l}1 \mathrm{M} \text { group } \\
(\mathrm{n}=46)\end{array}$ & $P$ value \\
\hline Major adverse cardiac events & $5(10.6)$ & $1(2.2)$ & 0.10 \\
\hline All-cause death & $0(0.0)$ & $0(0.0)$ & 1.00 \\
\hline Myocardial infarction & $0(0.0)$ & $0(0.0)$ & 1.00 \\
\hline Stent thrombosis & $0(0.0)$ & $0(0.0)$ & 1.00 \\
\hline TVR & $5(10.6)$ & $1(2.2)$ & 0.10 \\
\hline TLR & $4(8.5)$ & $1(2.2)$ & 0.18 \\
\hline Bleeding events & $16(34.0)$ & $12(26.1)$ & 0.41 \\
\hline Minor bleeding & $14(29.8)$ & $11(28.6)$ & 0.53 \\
\hline Major bleeding & $2(4.3)$ & $1(2.4)$ & 0.57 \\
\hline Composite endpoint & $21(44.7)$ & $13(28.3)$ & 0.10 \\
\hline
\end{tabular}

Data are given as $n(\%)$. DAPT, dual antiplatelet therapy; TLR, target lesion revascularization; TVR, target vessel revascularization.

of composite adverse, ischemic, and bleeding events during the 30-month follow-up period. Although no significant differences were observed, there was a tendency for fewer composite events in the $1 \mathrm{M}$ than standard group at the 30 -month follow-up time point $(28.3 \%$ vs. $44.7 \%$, respectively; $\mathrm{P}=0.41$ ). The incidence of bleeding events within 30 days was similar between the groups; however, the incidence of bleeding events from 30 days to 30 months tended to be lower in the $1 \mathrm{M}$ than standard group $(18.5 \%$ vs. $23.8 \%$; HR 0.66; 95\% CI 0.26, 1.65).

\section{Discussion}

In this study we compared the mid-term intrastent condition using OCT and the clinical outcomes at 30 months after R-ZES implantation between standard-duration and 1-month DAPT followed by prasugrel monotherapy. The main findings of this study are that: (1) at the 9-month follow-up, OCT revealed that the incidence of AITs, such as intrastent thrombus, was similar between the standard and $1 \mathrm{M}$ groups, despite the greater incidence of uncovered struts and smaller minimal stent area in the 1M group; (2) there were no significant differences in the efficacy and safety with regard to clinical endpoints of different DAPT durations when followed by prasugrel monotherapy.

\section{Short-Duration DAPT}

The optimal duration of DAPT after DES implantation has been debated for several decades. Prolonged DAPT is associated with a tradeoff between ischemic and bleeding risks. ${ }^{8}$ In the first-generation DES era, concern for very late stent thrombosis led to recommendations for longer ( $\geq 12$ months) DAPT; however, the introduction of secondgeneration DES and the widespread acceptance of optimal medical therapy have markedly decreased the incidence of stent thrombosis. ${ }^{3}$ Accordingly, recent studies have shown the efficacy and safety of shorter DAPT regimens. Furthermore, in accordance with the increased number of patients with a high bleeding risk who require PCI, it has become more important to avoid bleeding rather than ischemic events. The GLOBAL LEADERS trial was the first large-scale clinical trial seeking to explore the efficacy of very short-term (1-month) DAPT followed by ticagrelor monotherapy compared with the standard 12-month DAPT followed by aspirin monotherapy; this trial reported negative results in the prevention of all-cause mortality or new-onset myocardial infarction at 2 years after PCI. ${ }^{8}$ However, the ShorT and OPtimal Duration of Dual AntiPlatelet Therapy-2 Study (STOPDAPT-2), which compared the efficacy of 1-month DAPT followed by clopidogrel monotherapy with 12-month DAPT with aspirin and clopidogrel, reported a significantly lower rate of MACCE and bleeding events after the 1-month DAPT. ${ }^{3}$ In the present study we also demonstrated the non-inferiority of the 1-month DAPT regimen to the standard DAPT regimen for composite ischemic and bleeding events without an increase in intrastent thrombus. Therefore, a very short-term regimen of DAPT may be used to reduce bleeding events without increasing ischemic events. This study is one of the few studies supporting the non-inferiority of the very short-term (1-month) compared with standardduration DAPT.

\section{P2Y 12 Inhibitor Monotherapy}

The Clopidogrel versus Aspirin in Patients at Risk of Ischaemic Events (CAPRIE) trial showed that long-term administration of clopidogrel to patients with a primary atherosclerosis, as a cause of vascular diseases, is more effective in reducing the combined risk of ischemic vascular disease than long-term aspirin monotherapy. ${ }^{17}$ Subsequently, several randomized trials have been conducted to investigate the potential benefits of $\mathrm{P}_{2} \mathrm{Y}_{12}$ inhibitor monotherapy in reducing composite cardiovascular and bleeding events over aspirin., ${ }^{3,11}$

Prasugrel is a new-generation antiplatelet agent that shows more prompt, powerful, and consistent platelet inhibition than clopidogrel. ${ }^{9}$ The PRASugrel compared with clopidogrel For Japanese patIenTs with acute coronary syndrome (PRASFIT-ACS) study, which was designed to reduce the drug dose regimen (loading dose/maintenance dose: $20 / 3.75 \mathrm{mg}$ ) for Japanese patients who required PCI because they tended to have a higher mean age and lower body weight than Western patients, demonstrated that prasugrel was associated with a lower incidence of major adverse cardiac events and a lower risk of clinically serious bleeding in Japanese patients with acute coronary syndrome. ${ }^{9}$

Currently, prasugrel is a standard antiplatelet agent administered to Japanese patients who have undergone PCI; 


\section{(A) Composite endpoints}

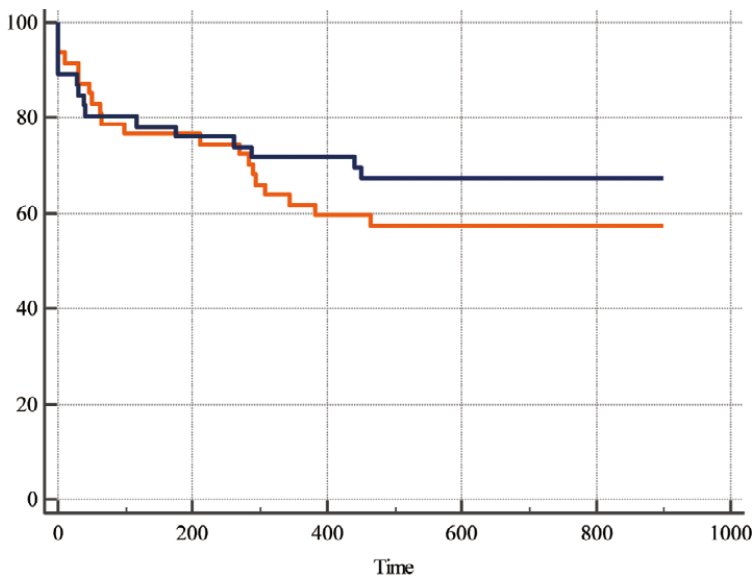

Log-rank $p=0.41$

(B) MACCE

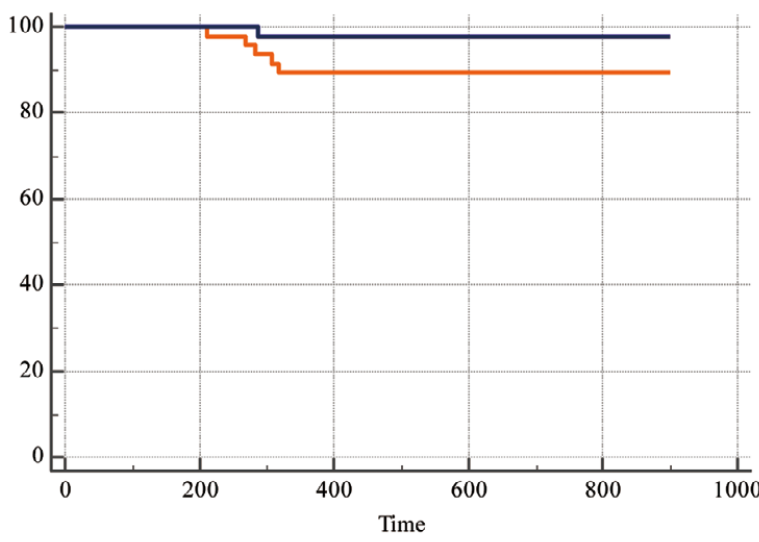

$\underline{\text { Log-rank } p=0.10}$

\section{(C) Bleeding events}

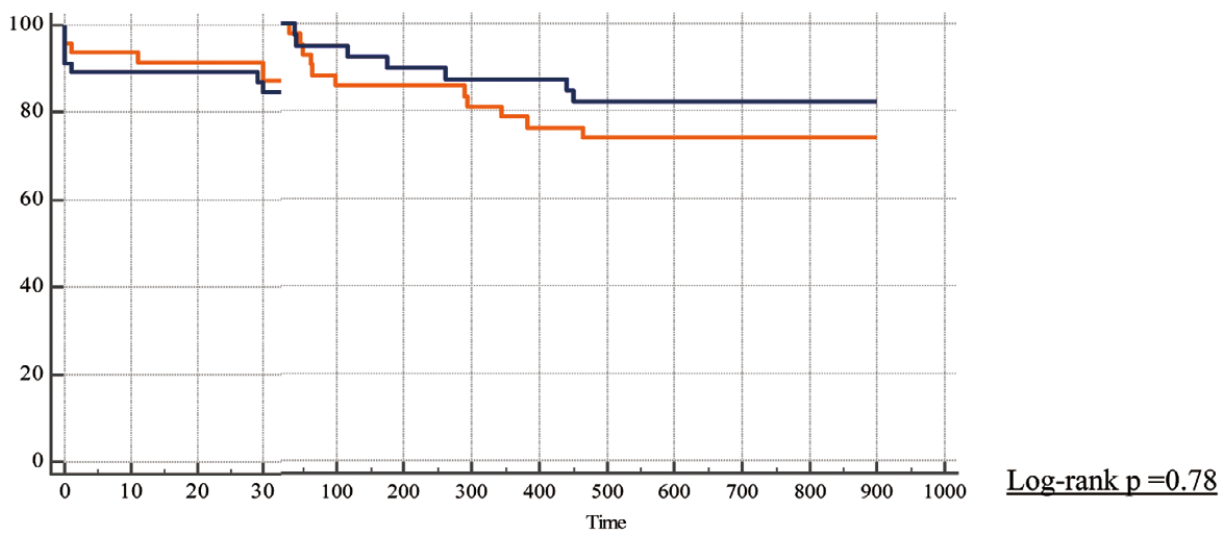

- Standard group $\quad-1 \mathrm{M}$ group

Figure 5. Results of Kaplan-Meier analysis. (A) Kaplan-Meier analysis of composite endpoints, including major adverse cardiovascular and cerebrovascular events (MACCE) and bleeding events, between patients receiving standard-duration dual antiplatelet therapy (DAPT) and those receiving 1-month DAPT followed by prasugrel (1M). (B) Comparison of MACCE between the 2 groups. (C) Comparison of bleeding events between the 2 groups. The incidence of bleeding events within the first 30 days was similar between the 2 groups, but the incidence from 30 days to 30 months tended to be lower in the $1 \mathrm{M}$ than standard group ( $18.5 \%$ vs. $23.8 \%$; hazard ratio 0.66 ; $95 \%$ confidence interval $0.26-1.65$ ). 
however, there are limited data regarding the efficacy and safety of prasugrel monotherapy, especially at the relatively low doses that is used in Japan. In the present study, patients in the $1 \mathrm{M}$ group stopped DAPT after 1 month, followed by prasugrel monotherapy that was continued for a long period of 30 months. We demonstrated the non-inferiority of prasugrel monotherapy to a standard regimen of DAPT. To the best of our knowledge, no other study to date has reported on prasugrel monotherapy after PCI for a long period; therefore, we believe that this study contributes to the literature by providing novel and valuable data.

\section{P2Y $_{12}$ Reaction Units}

Studies have reported that high and low PRU levels after PCI are significantly associated with ischemic and bleeding complications, respectively. ${ }^{18}$ The Platelet Reactivity in Patients with Drug Eluting Stent and Balancing Risk of Bleeding and Ischemic Event (PENDULUM) Registry suggested an association between high PRU levels and cardiovascular events in Japanese patients undergoing PCI. ${ }^{19}$ The PENDULUM Registry also demonstrated that MACCE were significantly increased in the high $(>208)$ than optimal (85-208) PRU group, and the trend was the same in patients with and without acute coronary syndrome. ${ }^{19}$ This study indicated that the mean PRU level in patients prescribed prasugrel was 163.5 , which was significantly lower than the PRU in patients prescribed clopidogrel (212.9). We also measured PRU levels in The PENDULUM study using VerifyNow P2Y12 assays immediately after PCI and 1, 3, and 9 months after PCI. Similar to the previous study, the mean PRU levels of the population in the present study at each time point was in the range 120-160, which is probably an optimal range of values to prevent ischemic and bleeding events. Thus, the incidence of ischemic events in the $1 \mathrm{M}$ group may be very low despite the very short DAPT duration, suggesting that maintaining PRU levels under control levels could prevent the development of ischemic events regardless of DAPT duration. Thus, PRU monitoring may be useful for risk stratification of MACCE, especially in patients receiving $\mathrm{P}_{2} \mathrm{Y}_{12}$ inhibitor monotherapy.

\section{OCT Findings and Stent Failure}

OCT provides high-resolution images of the coronary cross-section to evaluate vessel healing in response to stent implantation. Several studies have focused on identifying the most effective factor for predicting very late stent thrombosis using OCT and suggested that intrastent thrombus assessed using OCT could be a potential surrogate marker for the risk assessment of future stent thrombosis. ${ }^{15}$ Furthermore, delayed healing of stent struts, the presence of uncovered struts, and malapposed struts are associated with intrastent thrombus formation. Other studies have revealed that the independent predictors for intracoronary thrombus were a longer stent length, smaller stent diameter, and a greater number of uncovered struts. ${ }^{20}$ In the present study, notably, the mean stent area was smaller and the frequency of uncovered and malapposed struts was larger in the $1 \mathrm{M}$ group. From this aspect, the $1 \mathrm{M}$ group was more likely to develop stent failure; nevertheless, the incidence of AIT was similar between the $1 \mathrm{M}$ and standard groups, and the incidence of MACCE in the $1 \mathrm{M}$ group was very low $(2.2 \%)$. The latter could be attributed to the low PRU level of the $1 \mathrm{M}$ group. Therefore, when the PRU level was controlled at the optimal level, very short DAPT duration followed by $\mathrm{P}_{2} \mathrm{Y}_{12}$ inhibitor monotherapy is an acceptable antiplatelet strategy after second-generation DES implantation.

Furthermore, the frequency of AIT in the present study was much lower $(-1.5 \%)$ than in previous studies. Other studies have also stated that the frequency of thrombus was higher with first-generation DES (i.e., sirolimus-eluting stents) and lower with second-generation DES. 15,21,22 In the present study, we used a third-generation DES, namely R-ZES, which is a well-recognized DES with a low incidence of intrastent thrombus formation for all patients. Therefore, the incidence of AIT was very small in the present study.

\section{Study Limitations}

First, the present study was a single-center observational study with a small sample size. Ideally, the clinical events should have been the primary endpoint, but because of the small sample size we analyzed the presence of AITs as the primary endpoint. Therefore, this study may have been insufficiently powered to investigate the non-inferiority of very short DAPT relative to that of standard DAPT. Second, the study was not a randomized control trial and, therefore, there may be some selection bias. Indeed, some differences were recognized in patient background characteristics. For example, the PARIS thrombotic score was higher in the standard than $1 \mathrm{M}$ group. Hence, it is possible that many patients with poor conditions for stent implantation may have been included in the standard group. We did not include patients who required staged PCI in the 1M group, which may have led to a higher PARIS thrombotic score in the standard than $1 \mathrm{M}$ group. Third, we examined only the presence of AITs in the quantitative evaluation at the 9-month follow-up examination; however, further quantitative evaluations, such as neoatherosclerosis and peristrut low-intensity area, are required to predict late stent thrombosis in this third-generation DES era.

\section{Conclusions}

In conclusion, 1-month DAPT followed by prasugrel monotherapy was non-inferior to standard-duration DAPT in terms of intrastent thrombus formation, which may be a surrogate for late stent failure and composite adverse endpoints.

\section{Acknowledgments}

None.

\section{Sources of Funding}

This study did not receive any specific funding.

\section{Disclosures}

The authors declare that they have no conflicts of interest.

\section{IRB Information}

This study was approved by the Ethics Committee of Hyogo Prefectural Himeji Cardiovascular Center (Reference no. 2015-13).

\section{Data Availability}

The deidentified participant data will not be shared.

\section{References}

1. Mauri L, Kereiakes DJ, Yeh RW, Driscoll-Shempp P, Cutlip DE, Steg PG, et al. Twelve or 30 months of dual antiplatelet 
therapy after drug-eluting stents. $N$ Engl J Med 2014; 371: $2155-2166$.

2. Antman EM, Wiviott SD, Murphy SA, Voitk J, Hasin Y, Widimsky $\mathrm{P}$, et al. Early and late benefits of prasugrel in patients with acute coronary syndromes undergoing percutaneous coronary intervention: A TRITON-TIMI 38 (TRial to assess improvement in therapeutic outcomes by optimizing platelet inhibition with prasugrel-thrombolysis in myocardial infarction) analysis. J Am Coll Cardiol 2008; 51: 2028-2033.

3. Watanabe H, Domei T, Morimoto T, Natsuaki M, Shiomi H, Toyota T, et al. Effect of 1-month dual antiplatelet therapy followed by clopidogrel vs 12 -month dual antiplatelet therapy on cardiovascular and bleeding events in patients receiving PCI: The STOPDAPT-2 randomized clinical trial. JAMA 2019; 321: 2414-2427.

4. Nikolsky E, Stone GW, Kirtane AJ, Dangas GD, Lansky AJ, McLaurin B, et al. Gastrointestinal bleeding in patients with acute coronary syndromes: Incidence, predictors, and clinical implications: Analysis from the ACUITY (Acute Catheterization and Urgent Intervention Triage Strategy) trial. $J$ Am Coll Cardiol 2009; 54: $1293-1302$.

5. Kedhi E, Latib A, Abizaid A, Kandzari D, Kirtane AJ, Mehran R, et al. Rationale and design of the Onyx ONE global randomized trial: A randomized controlled trial of high-bleeding risk patients after stent placement with 1 month of dual antiplatelet therapy. Am Heart J 2019; 214: 134-141.

6. Hahn JY, Song YB, Oh JH, Chun WJ, Park YH, Jang WJ, et al. Effect of $\mathrm{P} 2 \mathrm{Y}_{12}$ inhibitor monotherapy vs dual antiplatelet therapy on cardiovascular events in patients undergoing percutaneous coronary intervention: The SMART-CHOICE randomized clinical trial. JAMA 2019; 321: 2428-2437.

7. Kim BK, Hong SJ, Cho YH, Yun KH, Kim YH, Suh Y, et al. Effect of ticagrelor monotherapy vs ticagrelor with aspirin on major bleeding and cardiovascular events in patients with acute coronary syndrome: The TICO randomized clinical trial. JAMA 2020; 323: 2407-2416.

8. Vranckx P, Valgimigli M, Jüni P, Hamm C, Steg PG, Heg D, et al. Ticagrelor plus aspirin for 1 month, followed by ticagrelor monotherapy for 23 months vs aspirin plus clopidogrel or ticagrelor for 12 months, followed by aspirin monotherapy for 12 months after implantation of a drug-eluting stent: A multicentre, open-label, randomised superiority trial. Lancet 2018; 392: 940 949.

9. Isshiki T, Kimura T, Ogawa H, Yokoi H, Nanto S, Takayama $\mathrm{M}$, et al. Prasugrel, a third-generation $\mathrm{P}_{2} \mathrm{Y}_{12}$ receptor antagonist, in patients with coronary artery disease undergoing elective percutaneous coronary intervention. Circ J 2014; 78: 2926-2934.

10. Costa F, van Klaveren D, James S, Heg D, Räber L, Feres F, et al. Derivation and validation of the predicting bleeding complications in patients undergoing stent implantation and subsequent dual antiplatelet therapy (PRECISE-DAPT) score: A pooled analysis of individual-patient datasets from clinical trials. Lancet 2017;
389: $1025-1034$.

11. Baber U, Dangas G, Cohen DJ, Gibson CM, Mehta SR, Angiolillo DJ, et al. Ticagrelor with aspirin or alone in high-risk patients after coronary intervention: Rationale and design of the TWILIGHT study. Am Heart J 2016; 182: 125-134.

12. Fujimoto W, Sawada T, Toba T, Takahashi Y, Miyata T, Oishi $\mathrm{S}$, et al. Comparison of the 9-month intra-stent conditions and 2-year clinical outcomes after Resolute zotarolimus-eluting stent implantation between 3-month and standard dual antiplatelet therapy. J Cardiol 2018; 72: 66-73.

13. Nagai H, Ishibashi-Ueda H, Fujii K. Histology of highly echolucent regions in optical coherence tomography images from two patients with sirolimus-eluting stent restenosis. Catheter Cardiovasc Interv 2010; 75: 961-963.

14. Oikawa Y, Yajima J, Costa MA, Matsuno S, Akabane M, Funada R, et al. Intravascular ultrasound, angioscopic and histopathological characterisation of heterogeneous patterns of restenosis after sirolimus-eluting stent implantation: Insights into potential "thromborestenosis" phenomenon. EuroIntervention 2010; 6: $380-387$

15. Nishio R, Shinke T, Otake H, Sawada T, Haraguchi Y, Shinohara $\mathrm{M}$, et al. Effect of cytochrome $\mathrm{P} 4502 \mathrm{C} 19$ polymorphism on target lesion outcome after drug-eluting stent implantation in Japanese patients receiving clopidogrel. Circ J 2012; 76: 2348-2355.

16. Mehran R, Rao SV, Bhatt DL, Gibson CM, Caixeta A, Eikelboom $\mathrm{J}$, et al. Standardized bleeding definitions for cardiovascular clinical trials: A consensus report from the Bleeding Academic Research Consortium. Circulation 2011; 123: 2736-2747.

17. Creager MA. Results of the CAPRIE trial: Efficacy and safety of clopidogrel. Clopidogrel versus aspirin in patients at risk of ischaemic events. Vasc Med 1998; 3: 257-260.

18. Breet NJ, van Werkum JW, Bouman HJ, Kelder JC, Ruven HJ, Bal ET, et al. Comparison of platelet function tests in predicting clinical outcome in patients undergoing coronary stent implantation. JAMA 2010; 303: 754-762.

19. Nakamura M, Kadota K, Takahashi A, Kanda J, Anzai H, Ishii $\mathrm{Y}$, et al. Relationship between platelet reactivity and ischemic and bleeding events after percutaneous coronary intervention in East Asian patients: 1-year results of the PENDULUM Registry. $J$ Am Heart Assoc 2020; 9: $\mathrm{e} 015439$.

20. Karimi Galougahi K, Maehara A, Shlofmitz R, Ben-Yehuda O, Mintz G, Stone G. Intracoronary optical coherence tomography 2018: Current status and future directions. JACC Cardiovasc Interv 2017; 10: 2473-2487.

21. Kim S, Kim JS, Shin DH, Kim BK, Ko YG, Choi D, et al. Comparison of early strut coverage between zotarolimus- and everolimus-eluting stents using optical coherence tomography. Am J Cardiol 2013; 111: 1-5.

22. Konishi A, Shinke T, Otake H, Takaya T, Nakagawa M, Inoue T, et al. Favorable vessel healing after Nobori biolimus A9-eluting stent implantation: 6- and 12-month follow-up by optical coherence tomography. Circ J 2014; 78: 1882-1890. 